\title{
QoS Provisioning for VoIP over Wireless Local Area Networks
}

\author{
Eng Hwee Ong, Jamil Y. Khan \\ School of Electrical Engineering and Computer Science \\ University of Newcastle, Australia, NSW 2308 \\ Email: enghwee.ong@studentmail.newcastle.edu.au, jamil.khan@ newcastle.edu.au
}

\begin{abstract}
With proliferation of the IEEE 802.11 WLAN and the emerging 802.11n standard, the WLAN is poised as a promising ubiquitous networking technology to support VoIP services, which is gaining popularity due to their high cost efficiency. However, the 802.11 WLAN is not designed to support delay sensitive traffic such as VoIP. This problem is magnified during a handover as user roam the WLAN network resulting in excessive handover latency and consequently packet loss. In addition, a 802.11 WLAN handover process is predominantly based on the physical layer detection without QoS considerations. This often causes overloading of access points and consequently all its associated connections would suffer from high delay, resulting in unacceptable QoS for the VoIP services. The former can be resolved by reducing handover latency to achieve seamless handover and the latter can be mitigated by employing link layer detection in the 802.11 WLAN handover process and having an appropriate admission control scheme. In this paper, we proposed an integrated load balancing scheme incorporating (i) QoSbased fast handover to support seamless handover by eliminating both detection and scanning phases from the 802.11 WLAN handover process; and (ii) soft admission control to protect QoS of existing voice connections when resources are low. This synergy guarantees service QoS during and after handover respectively. Simulations showed that our proposed integrated load balancing scheme is capable of providing seamless handover and QoS enhancements in terms of increased throughput, reduced packet loss and bounded delay when considering heterogeneous voice traffic of different packetization intervals. Particularly, our proposed scheme effectuate $Q o S$ balance of both delay and throughput which jointly optimize overall system utilization.
\end{abstract}

\section{INTRODUCTION}

Seamless mobility and packet voice communications are the driving forces of future wireless networks. The IEEE 802.11 WLAN permits connectivity of $11 \mathrm{Mbps}$ using the $802.11 \mathrm{~b}$ and high speed data rate of $54 M b p s$ using the $802.11 \mathrm{a} / \mathrm{g}$ necessary to support real-time voice services at low cost. The advent of 802.11n will further increase connection speed up to $600 \mathrm{Mbps}$. WLAN has experienced immense growth from providing coverage in enterprises to large scale deployment for hotspot coverage in hubs such as airports, hotels and campuses. Meanwhile, VoIP has been widely adopted for business communications and individuals using applications such as Skype for free global telephony. The unification of WLAN and VoIP known as voice over WLAN (VoWLAN) is compelling since VoIP can now leverage on pervasive 802.11 networks of high bandwidth for caller mobility. However, supporting voice traffic over WLAN poses numerous challenges such as network capacity, admission control and QoS provisioning.
Garg et al. [1] showed that it is crucial to determine the network capacity, in terms of the maximum number of simultaneous VoIP connections that can be supported by distributed coordination function (DCF) since its effective bandwidth is significantly reduced by inherent overheads which limit the maximum number of VoIP calls to a small number. Their study also suggested that admission control is vital for an infrastructure-based WLAN to protect existing VoIP connections. Zhai et al. [2] further found that WLAN attains maximum throughput and low delay when operating in unsaturated mode due to low collision probability, suggesting that admission control is a suitable strategy for real-time traffic due to its low bandwidth but strict delay requirements.

QoS provisioning in VoWLAN is challenging since the performance of their physical and medium access layers are incomparable to their wireline counterparts. One of the main challenges in VoWLAN is to support real-time VoIP connection with seamless handover since a WLAN of limited cell size results in frequent handover. VoIP requires one-way end-toend delay of less than $150 \mathrm{~ms}$ [3] but can tolerate some packet loss rate of up to $2 \%$ [4]. This implies that the total handover latency and packet loss should not exceed these bounds in order to sustain an undisruptive VoIP call of acceptable QoS. The total handover latencies include Layer 2 latency when station roams within the same IP subnet and Layer 3 latency (if necessary) when station roams across different IP subnet. We focus on minimizing Layer 2 handover latency which composes of detection delay, scanning delay, authentication delay and reassociation delay, where detection and scanning delays are the dominating cost [5], [6].

In this paper, we proposed an integrated load balancing scheme featuring (i) QoS-based fast handover to support seamless handover; and (ii) soft admission control to protect QoS of existing voice connections in VoWLAN, both by estimating critical network QoS parameters [7]. To the best of our knowledge, there is no prior research on load balancing scheme that guarantee service QoS and jointly optimize system utilization by considering QoS-based fast handover in conjunction with soft admission control. The remainder of the paper is organized as follows. Section II discusses related work. Section III describes our proposed integrated load balancing scheme and architecture. Section IV illustrates the simulation model. Section V presents the simulation results and Section VI concludes the paper. 


\section{RELATED WORK}

Various load metrics such as number of active connections, gross load, packet loss and throughput have been proposed in literature and it is one of the key elements in any load balancing scheme. For circuit-switched cellular networks such as GSM, load balancing is traditionally based on number of active calls per cell as its load metric since the load contributed by each user is the same. However, Bianchi et al. [8] showed that load balancing in packet-switched wireless networks such as WLAN can be improved by using additional "packet level" load metrics such as gross load which considers number of stations together with retransmission probability and packet loss. Bazzi et al. [9] developed a measurement-based call admission control to protect QoS of existing connections by denying incoming calls when resources are low. The merit of such measurement-based admission control is that it mitigates the difficulty to estimate the actual bandwidth occupancy of services due to data transmission rates variability and frame retransmissions. However, the parameters of their call admission control require tuning for different traffic mixes, hence not adaptive to dynamic network conditions.

Balachandran et al. [10] presented an adaptive load balancing solution where a centralized admission control server contains load information of all access points. It dictates station to associate with an access point that best suit its negotiated service level agreement. In the event when no suitable access points are available, the network would suggest roaming to further access points. However, this approach requires additional central server which increases network signaling overheads, creates bottleneck and prone to single point of failure. Velayos et al. [11] proposed a decentralized load balancing scheme using throughput per access point as their load metric. The access points are then classified in one of the three states, viz. underloaded which will accept any request, balanced which will only accept new calls or overloaded which will nominate a station for handover to an underloaded cell. However, the major pitfall of this scheme is that station will experience service outage during a handover since station first disassociate from an old access point and can only reassociate with an underloaded access point after some searching time has elapsed.

Our contributions differ from related works in three significant ways (i) we guarantee service QoS during handover by enabling seamless handover with QoS-based fast handover and guarantee service QoS after handover by operating network in unsaturated mode with a soft admission control; (ii) we exploit estimated critical network QoS parameters as criterion to select the best target network for handover and as load metric for soft admission control, both of which are adaptable to varying network conditions; and (iii) our distributed handover architecture provides network-assisted discovery compatible with IEEE 802.21 media independent handover infrastructure [12], thereby supports single transceiver stations, horizontal and vertical handovers. In addition, the terminal-oriented decision mechanism supports always best connected services.
III. Proposed Integrated Load Balancing Scheme AND ARCHITECTURE

The concept of our integrated load balancing scheme capitalized on both QoS-based fast handover to support seamless handover and soft admission control to protect QoS of existing voice connections by operating WLAN in unsaturated mode, thus preventing overloading of access points. Our proposed integrated load balancing scheme leverages on estimation of critical network QoS parameters, specifically, packet delay in this work. QoS parameters are in general non-stationary, however they can be considered as stationary when observed over a short period of time. We perform the bootstrap approximation in first stage to estimate the short-term stationary dynamic QoS parameters in an access point. We further account for the effects of non-stationary components in the second stage by performing the sequential Bayesian estimation with cumulative sum (CUSUM) monitoring in stations while listening to beacon broadcasts containing QoS parameter estimates. We refer readers to [7] for a more detailed description.

Accordingly, station would select the best access point according to their delay estimates which enabled us to obviate both detection and scanning phases of the 802.11 handover process, leading to significant Layer 2 handover latency reduction. The delay estimates are then augmented as load metric to devise a measurement-based soft admission control which is simple but yet effective as it considers dynamic network conditions prevalent in broadband WLANs. The key idea is to ensure that the delay threshold of an access point is not violated when accepting new connections which effectively protects QoS of existing connections by maintaining WLAN in an unsaturated mode. Soft admission control is important when considering heterogeneous voice traffic of different packetization intervals, since traditional hard admission control for homogeneous voice traffic that applies pre-determined network capacity directly as admission threshold is ineffective against such dynamic traffic.

We further advocate a distributed terminal-oriented, network assisted handover architecture suitable for VoWLAN system as depicted in Fig. 1. The shaded blocks refer to network entities while the unshaded blocks refer to terminal entities. Always best connected services which considers both network conditions and user preferences during a network selection can also be supported by completing the blocks in dashed lines. However, these are outside the scope of this work. Our proposed handover architecture can be triggered by two events, viz. initial access to network where station would choose the best network according to their service QoS (packet delay) requirement and handover when the network QoS (packet loss rate) exceeds $2 \%$ for the case of VoIP services. Soft admission control located in each station would arbitrate the prevailing traffic load and QoS in terms of delay between a source and target access points. Upon admission, station would perform (re)association with the selected target access point during (handover) initial access. Otherwise, the station would continue to monitor the packet loss rate of its associated 


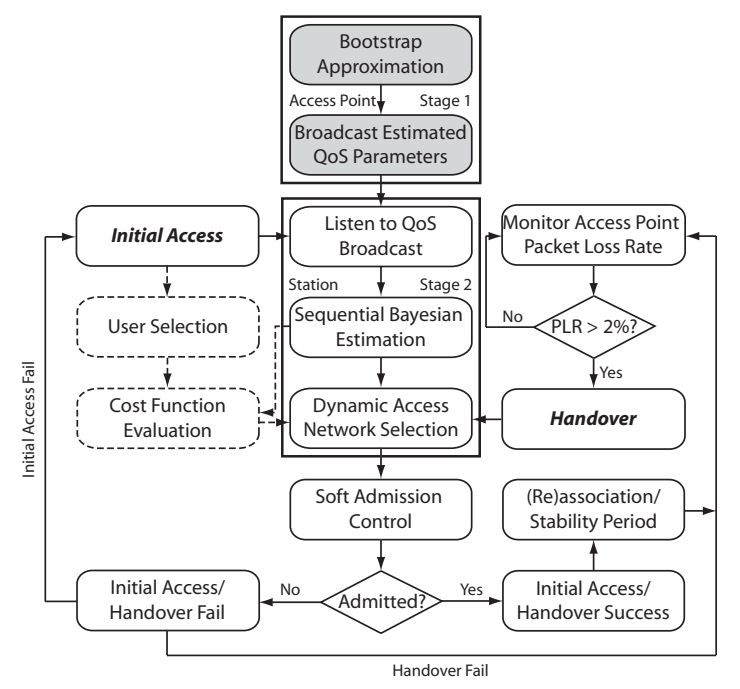

Fig. 1. Distributed terminal-oriented, network-assisted handover architecture.

access point when handover fails or listen to QoS broadcast should initial access fails. A stability period of $10 s$ is enforced before the other stations can make the next handover attempt to prevent the 'ping pong' effect.

The fundamental of our proposed handover architecture is network-assisted discovery such that source access point broadcasts measurement report of neighboring access points together with its own, compatible with the IEEE 802.21 media independent handover framework. As illustrated in Fig. 2, the estimated QoS parameters of an access point together with their channel number, location (cartesian coordinates) would be encapsulated in a packet as measurement report and transmitted to the access point controller (APC) periodically. The APC would then collect these measurement reports from every access point in their subnet. The consolidated measurement reports of the source and neighboring access points would finally be disseminated from APC using a location-based broadcast. This is motivated by the fact that it is not viable for stations to handover to a distant access point, making it unnecessary for station to monitor their network conditions. Since the locations of access points are usually fixed, it is feasible for an APC to maintain a location map information locally. Each APC can then exploit the location of source access point as center of circle with radius $r$ to filter out any access points that lie outside the circumference, thereby disseminating only measurement reports of three nearest neighboring access points for broadcast by source access point.

The advantage of our proposed handover architecture is twofold. First, it supports prevailing single transceiver station without any hardware modifications by requiring source access point to broadcast information of neighboring access points in addition to its own. Second, scanning procedures for handover decisions can be eliminated since station listening to the broadcast would be able to get information of prospective neighboring access points. Consequently, our total Layer 2 handover latency as illustrated in Fig. 3 is significantly reduced as both detection and scanning delay is obviated. We

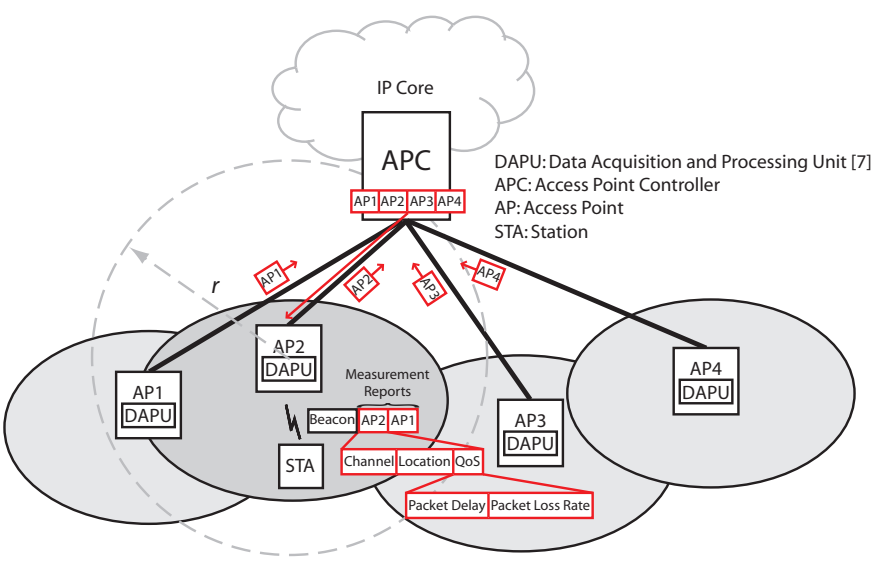

Fig. 2. Location-based broadcast in proposed handover architecture.

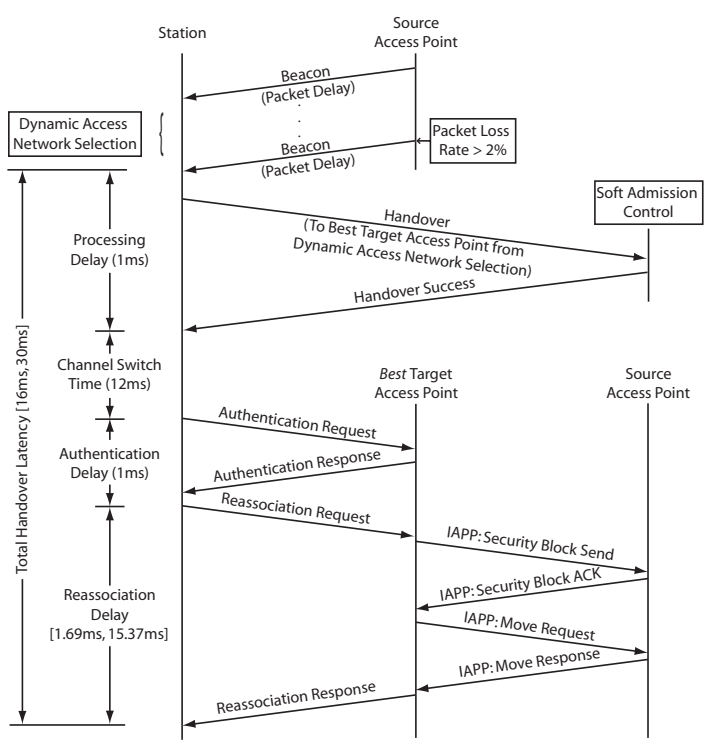

Fig. 3. Seamless handover process of integrated load balancing scheme.

exploited the fact that VoIP connections can tolerate some packet loss rate of $2 \%$ and utilize this as link layer detection to trigger handover. Since the best target access point is available from dynamic access network selection algorithm at the same instance, we do not incur any detection delay. Accordingly, it consists of 2-way handshake processing delay of typically $1 \mathrm{~ms}$ required by the soft admission control, average channel switch time of $12 \mathrm{~ms}$, authentication delay of less than $1 \mathrm{~ms}$ [13] and average reassociation delay of $15.37 \mathrm{~ms}$. We note that reassociation delay can be further reduced to $1.69 \mathrm{~ms}$ by applying neighbor graph technique [14]. Therefore, our total Layer 2 handover latency is approximately $16 \mathrm{~ms}$ to $30 \mathrm{~ms}$ as opposed to existing total handover latency of more than $1000 \mathrm{~ms}$ [6] when including link layer detection delay. Although, physical layer detection is widely deployed to exclude link layer detection delay but the total handover latency can be still as high as $420 \mathrm{~ms}$ [5].

\section{Simulation Model}

Without loss of generality, we used the wireline-to-wireless network topology as shown in Fig. 4 to investigate the effec- 


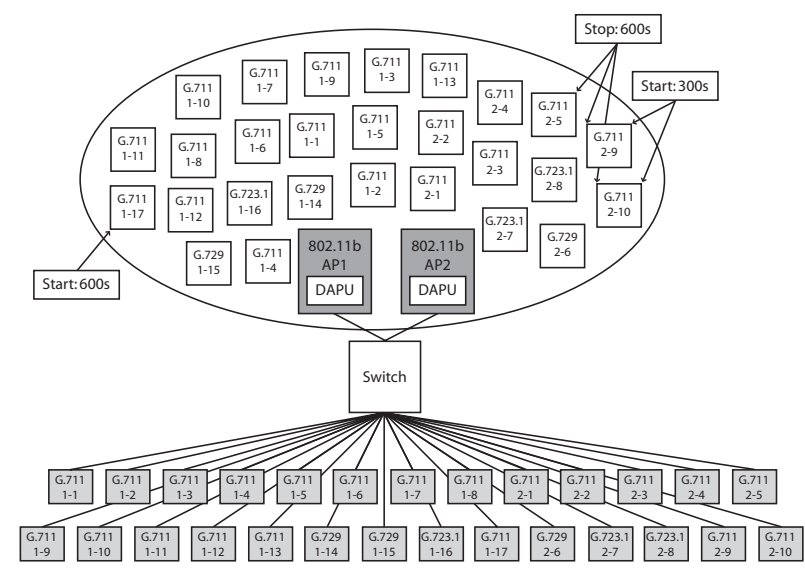

Fig. 4. VoWLAN simulation model with heterogeneous voice codecs.

tiveness of our integrated load balancing scheme in a typical hotspot scenario with two infrastructure-based $802.11 \mathrm{~b}$ access points operating with DCF at maximum data rate of $11 \mathrm{Mbps}$. We further subject our VoWLAN simulation to heterogeneous voice traffic of different packetization intervals. The simulation models were developed using OPNET ${ }^{\mathrm{TM}}$ Modeler $^{\circledR} 14.0$ with Wireless Module. We have modified the legacy DCF model to exclude scanning phase and integration with our custom model. We further assumed no hidden terminals and excluded RTS/CTS mechanism from our simulation. We have also incorporated MAC service data unit (MSDU) lifetime limit mechanism to discard MSDUs from the transmitter queue if they exceed the MSDU lifetime of $50 \mathrm{~ms}$ before successful transmission. This is favorable for delay-sensitive VoIP traffic since it minimizes unnecessary bandwidth consumption for transmission of ACK frames in attempt to acknowledge MSDUs that have exceeded their useful lifetime, hence mitigating any additional delay incurred while waiting for these ACK frames. All stations in our simulations are roaming capable to support handover events. Mobility model is not considered since we are interested in QoS-related handover rather than radio-related handover.

In order to focus on delay within each BSS, we assumed codec delay of $40 \mathrm{~ms}$, packetization delay of $20 \mathrm{~ms}$ at both sender and receiver and backbone network delay of $30 \mathrm{~ms}$. Therefore, the wireless network delay in both uplink and downlink should be less than $60 \mathrm{~ms}$ in order to meet the one-way end-to-end delay requirement of VoIP packets. VoIP packets are encoded using three popular voice codecs, viz. G.711, G.729 and G.723.1 with packetization interval of $10 \mathrm{~ms}, 20 \mathrm{~ms}$ and $30 \mathrm{~ms}$ respectively as shown in Table. I. We assumed that header compression is not used and added an additional 40 byte RTP/UDP/IP header to the payload. VoIP traffic are simulated with variable bit rate voice source using ON-OFF model where voice packets are only generated in the ON period. According to ITU [15], ON and OFF time can be approximated by an exponential distribution with mean of $1.004 \mathrm{~s}$ and $1.587 \mathrm{~s}$ respectively for speech activity of $39 \%$.

In our simulation, we initially introduced an unbalanced load of thirteen G.711, two G.729, one G.723.1 stations in
TABLE I

TRAFFIC GENERATION PARAMETERS.

\begin{tabular}{cccc}
\hline \hline $\begin{array}{c}\text { Traffic } \\
\text { Type }\end{array}$ & $\begin{array}{c}\text { Packet Size } \\
\text { (Bytes) }\end{array}$ & $\begin{array}{c}\text { Inter-arrival } \\
(\mathrm{ms})\end{array}$ & $\begin{array}{c}\text { Avg. Data Rate } \\
(\mathrm{kbps})\end{array}$ \\
\hline G.711 & 80 & 10 & 64 \\
G.729 & 20 & 20 & 8 \\
G.723.1 & 24 & 30 & 6.4 \\
\hline
\end{tabular}

BSS 1 and five G.711, one G.729, two G.723.1 stations in BSS 2. At time $300 \mathrm{~s}$, two G.711 connections were started in BSS 2. Subsequently at time $600 s$, one G.711 connection was started in BSS 1, while three G.711 connections were stopped in BSS 2. These discrete events induce imbalance traffic load during our simulation for evaluating the responsiveness of our integrated load balancing scheme under such dynamic network conditions. We note that no perturbations are injected after $600 s$ in order to observe the steady state performance.

Finally, we investigate the performance of our proposed integrated load balancing scheme from two critical aspects. First, we examine its QoS performance in terms of delay, throughput and packet loss of access points which reflects the capability of VoWLAN to support VoIP services. Second, we quantify the effect of load balancing on the overall system utilization by adopting the balance index introduced in [16] to reflect the used capacity in each access point. Suppose $x_{i}$ is the total throughput of access point $i$, then the balance index can be defined as,

$$
\mathrm{B}(x)=\left(\sum_{i} x_{i}\right)^{2} / n\left(\sum_{i} x_{i}^{2}\right),
$$

where $n$ is the number of access points over which the load will be redistributed. The balance index is a continuous function which is independent of scale. It is bounded between 0 and 1 such that it has a value of 1 when all access points have exactly the same throughput and a value of $1 / n$ when access points are extremely unbalance, which is 0 in the limit as $n \rightarrow \infty$.

\section{Simulation Results}

The simulation results presented include QoS performance of our integrated load balancing (iLB) scheme evaluated in terms of delay, throughput, packet loss and comparison with DCF which represent the case without load balancing. First, we investigate the average uplink and downlink delays associated with each access point. Each VoIP connection has duplex traffic which eventually results in higher downlink load, leading to the classical bottleneck at access point for infrastructure-based WLAN [17]. As such, the downlink delay is worse than its uplink delay as shown in Fig. 5. We noticed that access point 1 is overloaded while access point 2 is under-utilized for the case without iLB. This is predominantly due to physical layer detection of the existing 802.11 WLAN handover process which lacks QoS considerations. As a result, no handover is triggered since all stations are within good coverage region of their access points. Therefore, access point 1 has significantly higher uplink and downlink delays as compared to access point 2 . 


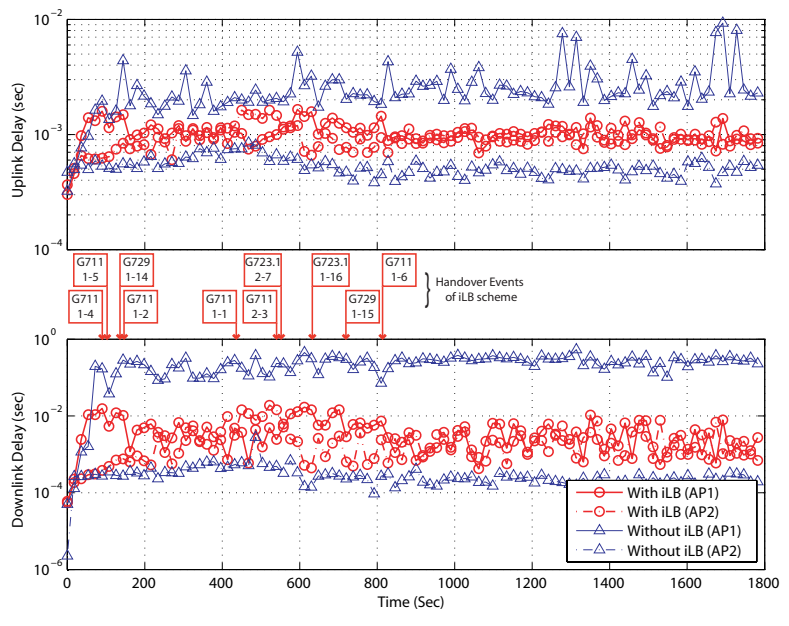

Fig. 5. Average uplink and downlink delays.

On the contrary, QoS-related handovers are observed with iLB as shown in Table. II, since it supports link layer detection which triggers a handover when packet loss rate of associated access point exceeds $2 \%$. This together with soft admission control effectively mitigate overloading of access point 1 . We note that a handover will only be triggered on the conditions that (i) packet loss rate of source access point is more than $2 \%$ and; (ii) there exists a target access point which can better meet the delay requirement of VoIP services. Finally, the handover attempt can only be completed if the target access point can still accept connections when subjected to soft admission control. As such, there will be no additional loss associated with a particular handover when sucessfully triggered and its service QoS shall be guaranteed after handover since WLAN will operate in unsaturated mode to protect QoS of existing connections. We remark that with iLB, the average uplink and downlink delays in both access points are less than $20 \mathrm{~ms}$ and balanced while for the case without iLB, an average downlink delay of up to $550 \mathrm{~ms}$ is experienced in access point 1 . Clearly with the introduction of iLB scheme, we are able to support VoIP connections of heterogeneous voice traffic with bounded and balanced average delays. The optimal balance of average uplink and downlink delays in both access points is achieved due to the fact that our estimated packet delay metric directly optimizes the expected packet delay, making it adaptive to dynamic network conditions.

Second, we examine uplink and downlink throughput. The former refers to aggregated throughput of access points and the latter refers to aggregated throughput of stations. Our simulation reveals that uplink throughput performance is similar as shown in Fig 6(a). This is not surprising as downlink is the bottleneck for infrastructure-based WLAN instead of uplink which is a consequence of the fairness in 802.11 DCF medium access scheme. Although, access points carry higher load than stations in a 2-way VoIP connection but both contend with the same priority. For downlink throughput, we noticed that iLB brings about and average enhancement of $6 \%$ as shown in Fig. 6(b). This enhancement is due to buffer overflow phenomenon in access point 1 for the case
TABLE II

HANDOVER EVENTS OF INTEGRATED LOAD BALANCING SCHEME. $\dagger$ two G.711 started in BSS 2 $\ddagger$ tone G.711 started in BSS 1 and three G.711 stopped in BSS 2

\begin{tabular}{|c|c|c|c|c|c|c|c|}
\hline \multirow{2}{*}{$\begin{array}{l}\text { Time } \\
(\mathrm{sec})\end{array}$} & \multirow{2}{*}{$\begin{array}{c}\text { Handover } \\
\text { STA }\end{array}$} & \multicolumn{3}{|c|}{ STAs in BSS 1} & \multicolumn{3}{|c|}{ STAs in BSS 2} \\
\hline & & G.711 & G.729 & G.723.1 & G.711 & G.729 & G.723.1 \\
\hline 0 & & 13 & 2 & 1 & 5 & 1 & 2 \\
\hline 91 & G711 1-4 & 12 & 2 & 1 & 6 & 1 & 2 \\
\hline 102 & G711 1-5 & 11 & 2 & 1 & 7 & 1 & 2 \\
\hline 135 & G729 1-14 & 11 & 1 & 1 & 7 & 2 & 2 \\
\hline 147 & G711 1-2 & 10 & 1 & 1 & 8 & 2 & 2 \\
\hline $300 \dagger$ & & 10 & 1 & 1 & 10 & 2 & 2 \\
\hline 436 & G711 1-1 & 9 & 1 & 1 & 11 & 2 & 2 \\
\hline 540 & G711 2-3 & 10 & 1 & 1 & 10 & 2 & 2 \\
\hline 551 & G723.1 2-7 & 10 & 1 & 2 & 10 & 2 & 1 \\
\hline $600 \ddagger$ & - & 11 & 1 & 2 & 7 & 2 & 1 \\
\hline 633 & G723.1 1-16 & 11 & 1 & 1 & 7 & 2 & 2 \\
\hline 717 & G729 1-15 & 11 & 0 & 1 & 7 & 3 & 2 \\
\hline 816 & G711 1-6 & 10 & 0 & 1 & 8 & 3 & 2 \\
\hline 1800 & - & 10 & 0 & 1 & 8 & 3 & 2 \\
\hline
\end{tabular}

without iLB, which is operating beyond its maximum capacity and hence experienced excessive packet loss. Although the downlink throughput enhancement is moderate, we note that both average uplink and downlink delays of access point 1 for the case without iLB as shown in Fig. 5 are not acceptable in terms of call quality for any VoIP connections. Moreover, a comparison of packet loss as shown in Fig. 6(c) reveals that, the total packets dropped from both access points for the case without iLB are eight times higher than for the case with iLB.

Finally, we showed in Fig. 7 that iLB effectuate optimal balance of network throughput in contrast to the case without iLB where network throughput are significantly imbalanced. We note that iLB delivers good steady state performance as there is no unnecessary handover when the throughput between access points are balanced. We attained optimal load balancing by virtue of our iLB scheme which supports soft admission control, adaptive to dynamic network conditions through the QoS parameters estimation. The self-adjusting soft admission control feature of our iLB scheme allows us to support heterogeneous voice traffic of different packetization intervals, which is not possible with the traditional hard admission control technique. We further remark that our iLB scheme offers balance from QoS perspective, particularly, it achieved QoS balance of both delay and throughput which jointly improves overall system utilization.

We have demonstrated the importance of integrated load balancing scheme in VoWLAN system. However, any derived benefit comes at a cost to both network and terminal which we would briefly discuss. For network, there will be no additional signaling overhead as measurement reports are appended to beacons, which are periodically broadcast by an access point to announce its existence. There would be some storage and communication overheads for updating these measurement reports. However, this would not impose heavy loads since location-based broadcast is restricted to only three nearest neighboring access points. For terminal, we consider computational complexity which would manifest as power consumption. Although, our proposed scheme requires additional computational to perform network selection but we expect it to be minimal since our algorithm has linear time complexity of $O(n)$. Moreover, the exclusion of scanning phases in our QoS-based fast handover scheme helps to offset this incremental computational cost. 


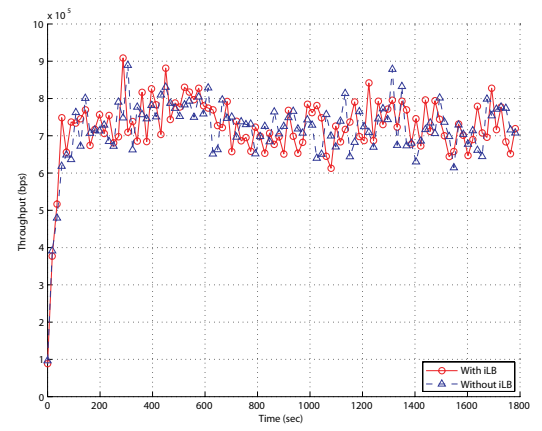

(a) Uplink throughput.

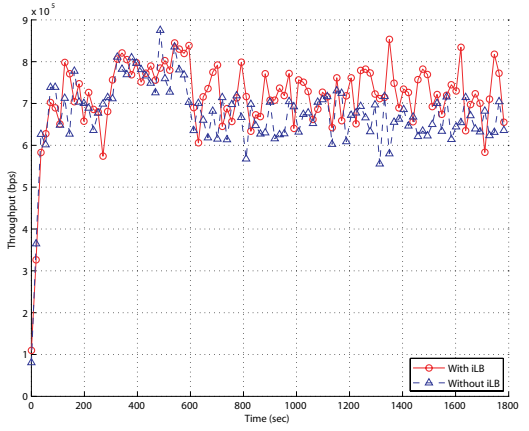

(b) Downlink throughput.

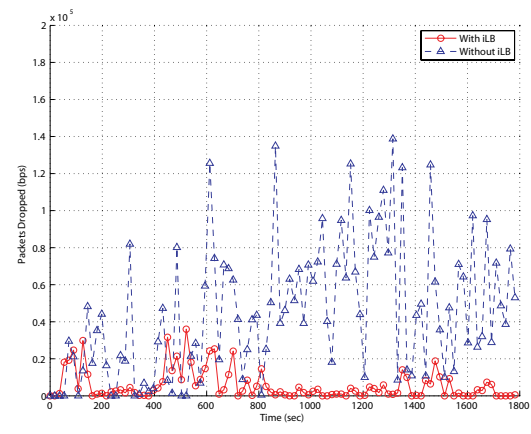

(c) Packet loss.

Fig. 6. Throughput and packet loss.

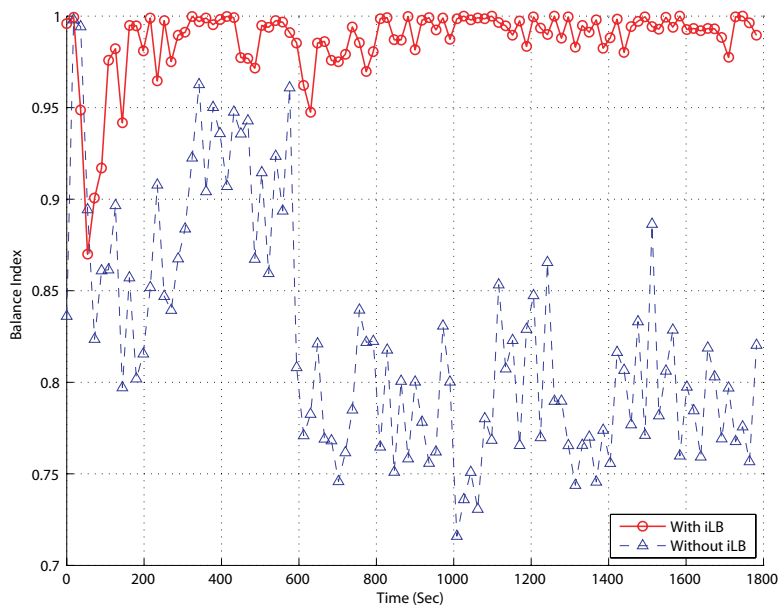

Fig. 7. Balance index of network throughput.

\section{CONCLUSION}

We presented an integrated load balancing (iLB) scheme that leverages on QoS-based fast handover to provide seamless handover and soft admission control to protect QoS of existing voice connections when resources are low. By means of estimating critical QoS parameters, we are able to eliminate both detection and scanning phases from the 802.11 WLAN handover process and devise a robust soft admission control to support heterogeneous voice traffic of different packetization intervals, otherwise not conceivable with hard limiting approaches. We showed by induction that our iLB scheme is able to support seamless handover with total Layer 2 handover latency of $16 \mathrm{~ms}$ to $30 \mathrm{~ms}$. We further demonstrated by simulation that a bounded average delay of less than $20 \mathrm{~ms}$ is achievable in both directions. These satisfy the stringent QoS requirements of real-time VoIP connections for VoWLAN system. In summary, iLB offers four main benefits, viz. (i) delay guarantee of VoIP stations during handover with fast handover; (ii) delay guarantee of VoIP stations after handover with soft admission control; (iii) throughput gain of $6 \%$ on the downlink which translates to eightfold enhancement in terms of packet loss; and (iv) optimal QoS balance of both delay and throughput for a multi-access point network which jointly improves overall system utlization.

\section{REFERENCES}

[1] S. Garg and M. Kappes. An experimental study of throughput for UDP and voIP traffic in IEEE 802.11b networks. 2003. WCNC 2003. 2003 IEEE Wireless Communications and Networking, 3:1748-1753, March 2003.

[2] H. Zhai, X. Chen, and Y. Fang. How well can the IEEE 802.11 wireless LAN support quality of service? IEEE Transactions on Wireless Communications, 4(6):3084-3094, November 2005.

[3] ITU-TG.114. One-way transmission time. 2003.

[4] C. Shim, L. Xie, B. Zhang, and C. Sloane. How delay and packet loss impact voice quality in voip. Technical report, Qovia, Inc., December 2003.

[5] A. Mishra, M. Shin, and W. Arbaugh. An empirical analysis of the ieee 802.11 mac layer handoff process. SIGCOMM Comput. Commun. Rev., 33(2):93-102, 2003.

[6] H. Velayos and G. Karlsson. Techniques to reduce the IEEE $802.11 \mathrm{~b}$ handoff time. In Communications, 2004 IEEE International Conference on, volume 7, pages 3844-3848, June 2004.

[7] E. H. Ong and J. Y. Khan. Dynamic access network selection with QoS parameters estimation: A step closer to ABC. In Vehicular Technology Conference, 2008. VTC Spring 2008. IEEE, pages 2671-2676, Marina Bay, Singapore, May 2008.

[8] G. Bianchi and I. Tinnirello. Improving load balancing mechanisms in wireless packet networks. In Communications, 2002. ICC 2002. IEEE International Conference on, volume 2, pages 891-895, 2002.

[9] A. Bazzi, M. Diolaiti, and G. Pasolini. Measurement based call admission control strategies in infrastructured IEEE 802.11 WLANs. In Personal, Indoor and Mobile Radio Communications, 2005. PIMRC 2005. IEEE 16th International Symposium on, volume 3, pages $2093-$ 2098, September 2005.

[10] A. Balachandran, P. Bahl, and G. M. Voelker. Hot-spot congestion relief in public-area wireless networks. In Mobile Computing Systems and Applications, 2002. Proceedings Fourth IEEE Workshop on, pages 7080, 2002.

[11] H. Velayos, V. Aleo, and G. Karlsson. Load balancing in overlapping wireless LAN cells. In Communications, 2004 IEEE International Conference on, volume 7, pages 3833-3836, June 2004.

[12] IEEE-P802.21/D10.0. Draft ieee standard for local and metropolitan area networks: Media independent handover services. April 2008.

[13] I. Ramani and S. Savage. Syncscan: practical fast handoff for 802.11 infrastructure networks. In INFOCOM 2005. 24th Annual Joint Conference of the IEEE Computer and Communications Societies. Proceedings IEEE, volume 1, pages 675-684, March 2005.

[14] A. Mishra, M. Shin, and W. A. Arbaush. Context caching using neighbor graphs for fast handoffs in a wireless network. In INFOCOM 2004. Twenty-third AnnualJoint Conference of the IEEE Computer and Communications Societies, volume 1, March 2004.

[15] ITU-TP.59. Artificial conversational speech. 1993.

[16] D. Chiu and R. Jain. Analysis of the increase and decrease algorithms for congestion avoidance in computer networks. Comput. Netw. ISDN Syst., 17(1):1-14, 1989.

[17] S. Shin and H. Schulzrinne. Balancing uplink and downlink delay of voip traffic in wlans using adaptive priority control (apc). In QShine '06: Proceedings of the 3rd international conference on Quality of service in heterogeneous wired/wireless networks, page 41, New York, NY, USA, 2006. ACM. 